TRANSACTIONS OF THE

AMERICAN MATHEMATICAL SOCIETY

Volume 360, Number 10, October 2008, Pages 5145-5154

S 0002-9947(08)04605-9

Article electronically published on May 27, 2008

\title{
POLYNOMIALS WITH COEFFICIENTS FROM A FINITE SET
}

\author{
PETER BORWEIN, TAMÁS ERDÉLYI, AND FRIEDRICH LITTMANN
}

Abstract. In 1945 Duffin and Schaeffer proved that a power series that is bounded in a sector and has coefficients from a finite subset of $\mathbb{C}$ is already a rational function. Their proof is relatively indirect. It is one purpose of this paper to give a shorter direct proof of this beautiful and surprising theorem.

This will allow us to give an easy proof of a recent result of two of the authors stating that a sequence of polynomials with coefficients from a finite subset of $\mathbb{C}$ cannot tend to zero uniformly on an arc of the unit circle.

Another main result of this paper gives explicit estimates for the number and location of zeros of polynomials with bounded coefficients. Let $n$ be so large that

$$
\delta_{n}:=33 \pi \frac{\log n}{\sqrt{n}}
$$

satisfies $\delta_{n} \leq 1$. We show that any polynomial in

$$
K_{n}:=\left\{\sum_{k=0}^{n} a_{k} z^{k}:\left|a_{0}\right|=\left|a_{n}\right|=1 \text { and }\left|a_{k}\right| \leq 1\right\}
$$

has at least

$$
8 \sqrt{n} \log n
$$

zeros in any disk with center on the unit circle and radius $\delta_{n}$.

\section{INTRODUCTION}

This paper deals with questions of how polynomials with coefficients from a finite subset of $\mathbb{C}$ behave near and on the unit circle. We investigate two related problems; first we ask how many zeros such a polynomial has near the unit circle, and secondly we ask how fast sequences of these polynomials can converge to zero.

The answer to the first question turns out to be 'quite a lot'; in contrast, in answer to the second question, as was shown in [3], sequences of such polynomials cannot converge to zero uniformly on any arc of the unit circle.

Figure 1 shows the zeros of all polynomials of degree 18 with coefficients from the set $\{-1,+1\}$. The structure is quite striking and suggests a subtlety that one might not initially expect. Some of the subtleties of such sets are explored by Odlyzko and Poonen in [13. See also [1, 4] and 14.

Figure 2 shows the zeros of polynomial of degree 800 with random coefficients from the set $\{-1,+1\}$.

In this paper we prove new estimates for the number of zeros of polynomials with bounded coefficients near the unit circle, and we give a new proof of the result

Received by the editors June 8, 2005 and, in revised form, February 15, 2006.

2000 Mathematics Subject Classification. Primary 30B30; Secondary 11C08, $30 \mathrm{C} 15$.

Key words and phrases. Zeros, rational functions, Duffin-Schaeffer Theorem, Littlewood polynomials. 


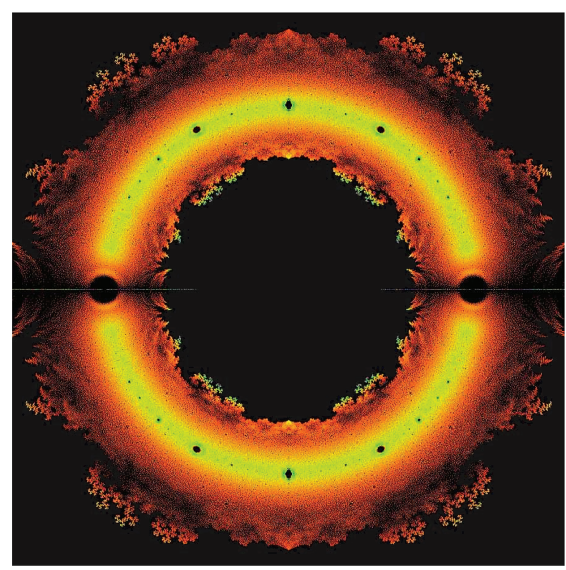

Figure 1. Zeros of all polynomials of degree 18 with coefficients from the set $\{-1,+1\}$.

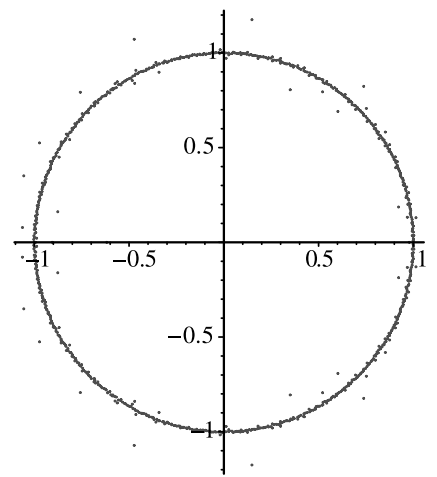

Figure 2. Zeros of a polynomial of degree 800 with coefficients from $\{-1,+1\}$.

from [3] using a theorem of Duffin and Schaeffer [7] which sheds some light on the reasons why uniform convergence to zero cannot occur on any arc of the unit circle.

We define a class of polynomials by

$$
K_{n}:=\left\{f(x):=\sum_{j=0}^{n} a_{j} x^{j}:\left|a_{0}\right|=\left|a_{n}\right|=1 \text { and }\left|a_{j}\right| \leq 1\right\} .
$$

Throughout this paper we use the notation $D(z, r)$ for the open disk centered at $z \in \mathbb{C}$ with radius $r$. Define

$$
\delta_{n}:=33 \pi \frac{\log n}{\sqrt{n}}
$$

In Section 2 we prove the following estimate.

Theorem 1. Let $\left|z_{0}\right|=1$. If $n$ is so large that $\delta_{n} \leq 1$, then every $P \in K_{n}$ has at least $8 \sqrt{n} \log n$ zeros in the open disk $D\left(z_{0}, \delta_{n}\right)$. 
Associated with a sequence $\left(p_{j}\right)_{j=1}^{\infty}$ of distinct Littlewood polynomials, that is, polynomials with coefficients in $\{ \pm 1\}$, let

$$
S:=\left\{z \in \mathbb{C}: p_{j}(z)=0 \text { for some } j\right\} .
$$

Theorem 1 implies in particular that the unit circle is a subset of the closure of $S$, a result that can also be deduced from Theorem 3.4.1 in [12] due to P. Erdös and P. Turán.

We now investigate the behavior of sequences of polynomials on arcs of the unit circle.

Theorem 2 (cf. [3]). Let $A$ be an arc on the unit circle. If $\left(p_{n}\right)$ is a sequence of polynomials with coefficients from a finite subset of $\mathbb{C}$, then $\left(p_{n}\right)$ cannot tend to zero uniformly on A.

We show that this theorem, originally obtained by the two first-named authors, can be deduced from a theorem of Duffin and Schaeffer [7]. This approach is less technical than the original proof and sheds some light on the reasons why uniform convergence to zero cannot occur.

To prove Theorem 2 assume that a sequence $\left(p_{n}\right)$ of polynomials having only finitely many distinct coefficients tends to zero uniformly on an arc of the unit circle. This means that we can find a subsequence $\left(p_{n_{k}}\right)$ so that

$$
\sum_{k=1}^{\infty} p_{n_{k}}(z)
$$

converges uniformly for $z$ on the arc. Now choose an increasing sequence of positive integers $\lambda_{k}$ growing rapidly enough so that

$$
f(z):=\sum_{k=1}^{\infty} p_{n_{k}}(z) z^{\lambda_{k}}
$$

will satisfy Fabry's gap condition. Note that by construction this power series is bounded in a sector of the unit circle, has finitely many distinct coefficients, and by Fabry's gap theorem the unit circle is its natural boundary. However, the following theorem, due to Duffin and Schaeffer, shows that a power series of this form cannot exist.

Theorem 3 (cf. 7]). Let $f(z)$ be a power series whose coefficients are taken from a finite subset of $\mathbb{C}$. If $f(z)$ is bounded in a sector of the open unit disk, then it is a rational function.

The theorem of Duffin and Schaeffer is a generalization of a result of Szegö [17. who proved in 1922 that a power series $f$ whose coefficients assume only finitely many values and which can be extended analytically beyond the unit circle is already a rational function.

When passing from power series that have an analytic continuation beyond the unit circle to power series that are bounded in a sector, the condition that the coefficients of the power series are taken from a finite subset of $\mathbb{C}$ becomes crucial. A power series with integer coefficients that has an analytic continuation beyond the unit circle is already a rational function; in contrast, Duffin and Schaeffer give the example

$$
\sum_{n=1}^{\infty}(1-z)^{n} z^{n !}
$$


of a function with integer coefficients which is bounded in the sector of the unit circle $-\pi / 4 \leq \arg z \leq \pi / 4$ but which has the unit circle as its natural boundary.

Duffin and Schaeffer obtain their result as a corollary of an investigation of the behavior of entire functions of finite exponential type $\tau<\pi$ which are bounded on certain real sequences of points. By interpreting the coefficients of $f$ as values of such an entire function at the positive integers, they arrive at Theorem 3 .

It is an open problem whether Theorem 2 remains true after replacing arcs on the unit circle with sets of positive measure on the unit circle. The proof of Theorem 2 would remain valid if an analogue of Theorem 3 for sets of positive measure were true. In order to gain a better understanding of Theorem 3, we give a direct proof in Section 3 .

It is an exercise to characterize rational functions with coefficients from any fixed finite subset of $\mathbb{C}$.

Theorem 4. If $f$ is a rational function with coefficients from $\{0,1\}$ in their Taylor series expansion at the origin, then

$$
f(z)=p_{m}(z)+z^{m+1} \frac{q_{n}(z)}{1-z^{n+1}}
$$

where $p_{m}$ is a polynomial with coefficients from $\{0,1\}$ of degree at most $m$ and $q_{n}$ is a polynomial with coefficients from $\{0,1\}$ of degree at most $n$. If $f$ has no zeros in the unit disk, then all roots of $\left(1-z^{n+1}\right) p_{m}(z)+z^{m+1} q_{n}(z)$ are roots of unity.

Proof. Note that if $f$ is a rational function, then it is a quotient of two polynomials with rational coefficients. Since $f(1 / 2)$ is rational, the binary expansion of $f(1 / 2)$ (which is just the sequence of coefficients of $f$ ) is ultimately periodic. The result follows immediately.

There is a similar result if the coefficients of $f$ come from a finite set of complex numbers. See Pólya and G. Szegö, Volume 2, P. 158, Ex. 158.

\section{ZEROS OF POLYNOMIALS WITH BOUNDED COEFFICIENTS}

In this section we prove Theorem [1. The proof follows from the combination of the two results below. The first one is a difficult result of Erdős and P. Turán [9]; the second one is a consequence of Jensen's Formula observed in 8 .

Recall that $K_{n}$ is the class of polynomials of degree $n$ having a constant and leading term of absolute value 1 and all coefficients bounded by 1 in absolute value.

Lemma 1 (Erdős and Turán [9]). If the zeros of

$$
P(z):=\sum_{k=0}^{n} a_{k} z^{k}
$$

are denoted by

$$
z_{\nu}=r_{\nu} \exp \left(i \varphi_{\nu}\right), \quad r_{\nu}>0, \quad \varphi_{\nu} \in[0,2 \pi), \quad \nu=1,2, \ldots, n,
$$

then for every $0 \leq \alpha<\beta \leq 2 \pi$ we have

$$
\left|\sum_{\nu \in I(\alpha, \beta)} 1-\frac{\beta-\alpha}{2 \pi} n\right|<16 \sqrt{n \log R},
$$

where $R:=\left|a_{0} a_{n}\right|^{-1 / 2}\left(\left|a_{0}\right|+\left|a_{1}\right|+\cdots+\left|a_{n}\right|\right)$ and $I(\alpha, \beta):=\left\{\nu: \alpha \leq \varphi_{\nu} \leq \beta\right\}$. 
Lemma 2. Let $\alpha$ be a number in $(0,1)$. Every polynomial in $K_{n}$ has at most $(2 / \alpha) \log (2 / \alpha)$ zeros in the open disk $D(0,1-\alpha)$ centered at the origin with radius $1-\alpha$ and outside the open disk $D\left(0,(1-\alpha)^{-1}\right)$ centered at the origin with radius $(1-\alpha)^{-1}$.

Proof. We give a sketch of the proof from 8]. Let $p \in K_{n}$. The inequality $|z|(1-|z|)^{-1}<1$ for $|z|<1 / 2$ implies $|p(z)|>1-|z|(1-|z|)^{-1}>0$, hence we may assume that $\alpha \in(0,1 / 2]$.

Any $p \in K_{n}$ satisfies $|p(z)| \leq(1-|z|)^{-1}$ for $|z|<1$. Since $p(0) \neq 0$, Jensen's formula (Section 4.2 of [2]) applied to the zeros $a_{1}, \ldots, a_{m}$ of $p$ in the disk $D(0,1-\alpha / 2)$ (the zeros are listed according to their multiplicity) implies

$$
\sum_{k=1}^{m} \log \frac{1-\alpha / 2}{\left|a_{k}\right|}=\frac{1}{2 \pi} \int_{0}^{2 \pi} \log \left|p\left((1-\alpha / 2) e^{i \theta}\right)\right| d \theta \leq \log \frac{2}{\alpha}
$$

For $|z|<1-\alpha$ we have $\alpha / 2 \leq \log ((1-\alpha / 2) /|z|)$, and hence, omitting the terms in (2.1) for which $\left|a_{k}\right| \geq 1-\alpha$, we obtain for the number $M$ of zeros of $p$ in $D(0,1-\alpha)$ that

$$
\frac{M \alpha}{2} \leq \log \frac{2}{\alpha}
$$

The assertion about the number of zeros of $p$ outside of $D\left(0,(1-\alpha)^{-1}\right)$ follows from the observation that this number equals the number of zeros of $z^{n} p(1 / z) \in K_{n}$ in $D(0,1-\alpha)$.

We are now ready to give the

Proof of Theorem 1. Let $z_{0}:=e^{i \varphi}$. For $\gamma>0$ consider the sector

$$
S\left(z_{0}, \gamma\right):=\left\{r e^{i \theta}: 0 \leq r<\infty, \varphi-\gamma<\theta<\varphi+\gamma\right\} .
$$

Let $n$ be so large that the inequality

$$
\gamma_{n}:=\frac{32 \pi \log n}{\sqrt{n}} \leq 1
$$

is satisfied. Let $P \in K_{n}$, and let $m$ be the number of zeros of $P$ in $\mathbb{C} \backslash S\left(z_{0}, \gamma_{n}\right)$. It follows from Lemma 1 that

$$
m-\frac{2 \pi-2 \gamma_{n}}{2 \pi} n \leq 16 \sqrt{n \log n},
$$

that is,

$$
m \leq n-\frac{\gamma_{n} n}{\pi}+16 \sqrt{n \log n},
$$

hence $m \leq n-16 \sqrt{n} \log n$. Therefore $P$ has at least $16 \sqrt{n} \log n$ zeros in the sector $S\left(z_{0}, \gamma_{n}\right)$. Now we use Lemma 2 with $\alpha:=n^{-1 / 2}$ to obtain at least $8 \sqrt{n} \log n$ zeros in the intersection of the sector $S\left(z_{0}, \gamma_{n}\right)$ and the annulus $1-1 / \sqrt{n}<|z|<$ $(1-1 / \sqrt{n})^{-1}$. Since the disk with center at $z_{0}$ and radius $\delta_{n}:=33 \pi \log n / \sqrt{n}$ contains this intersection, the statement of Theorem 1 follows. 


\section{POWER SERIES BOUNDED IN A SECTOR}

The proof of Theorem 3 given here combines ideas of Dienes [6], Szegö [17] and Duffin and Schaeffer [7. It rests on two facts for power series with bounded coefficients, namely, a series which satisfies the assumptions of Theorem 3 can be represented as a sum of two power series, one with nice analytic behavior, the other with coefficients that go to zero, and secondly, coefficients of power series with a continuation beyond the unit circle behave almost like coefficients of rational functions:

Lemma 3 (cf. Lemma 1 on page 504 of [6]). Let $f$ be a power series which is bounded in a sector and has bounded coefficients. Then $f=f_{1}+f_{2}$, where $f_{1}$ is a power series with bounded coefficients having an analytic continuation beyond the unit circle, and $f_{2}$ is a power series whose coefficients go to zero.

Lemma 4 (cf. Satz 1 in [17]). Let $g(z)=b_{0}+b_{1} z+\cdots$ be a power series with bounded coefficients which can be analytically continued beyond the unit circle at at least one point. Then for all $\varepsilon>0$ there exist a positive integer $q$ and complex numbers $\gamma_{0}, \ldots, \gamma_{q-1}$ such that for all $n \geq 0$ the inequality

$$
\left|\gamma_{0} b_{n}+\cdots+\gamma_{q-1} b_{n+q-1}+b_{n+q}\right|<\varepsilon
$$

holds.

We require some notation. Let $\tau$ be an angle with $0<\tau<\pi / 2$, let $\delta>0$, and let $r$ be a radius with $r \geq 1-\delta$. (For the proof of Lemma 3 we will consider $r$ with $r \leq 1$; for the proof of Lemma 4 we will allow $r>1$.) Denote by $\Gamma_{\tau}(\delta, r)$ the curve

$$
\begin{aligned}
& |z|=1-\delta \text { for } \tau<\arg z<2 \pi-\tau, \\
& 1-\delta \leq|z|<r \text { for } \arg z \in\{\tau,-\tau\}
\end{aligned}
$$

and denote by $A_{\tau}(r)$ the arc given by $|z|=r$ and $-\tau \leq \arg z \leq \tau$. Finally, denote by $K_{\tau}(\delta, r)$ the contour consisting of the union of $\Gamma_{\tau}(\delta, r)$ and $A_{\tau}(r)$, and denote by $C_{\tau}(\delta, r)$ the image of $K_{\tau}(\delta, r)$ under the transformation $z \mapsto z^{-1}$. Note that $C_{\tau}(0, r)$ is a curve in the closed unit disk with a positive proportion inside the open unit disk.

Proof of Lemma 3. The proof uses an idea of Duffin and Schaeffer (cf. page 151 of [7). We may assume that $f$ is bounded in a sector given by $|z|<1$ and $-\tau \leq$ $\arg z \leq \tau$, and that $f(0)=0$ holds. For $r$ with $1-\delta<r \leq 1$ we let

$$
f_{r, 1}(z):=\frac{1}{2 \pi i} \int_{\Gamma_{\tau}(\delta, r)} \frac{f(\zeta)}{\zeta-z} d \zeta
$$

and note that $f_{r, 1}$ is analytic on $\mathbb{C} \backslash \Gamma_{\tau}(\delta, r)$. In particular, $f_{1,1}$ is regular on the arc $A_{\tau}(1)$. We define for $r<1$

$$
f_{r, 2}(z):=\frac{1}{2 \pi i} \int_{A_{\tau}(r)} \frac{f(\zeta)}{\zeta-z} d \zeta
$$

and denote by $a_{r, n}$ the coefficients of the power series expansion of $f_{r, 2}$ at the origin. Consider for $|z|<1$ the integral

$$
F(z):=\int_{0}^{z} \frac{f(\zeta)}{\zeta} d \zeta .
$$


Assume that $r<1$. Computing $f_{r, 2}^{(n)}(0)$ from (3.3) , letting $\zeta=r e^{i \theta}$ and noting that $z^{-1} f(z) d z=d F(z)$ shows

$$
a_{r, n}=\frac{r^{-n}}{2 \pi i} \int_{-\tau}^{\tau} e^{-i n \theta} d F\left(r e^{i \theta}\right)
$$

where the integral is a Lebesgue-Stieltjes integral with integration variable $\theta$. We want to show that, as $r \rightarrow 1-, a_{r, n}$ converges to $a_{1, n}$ for all $n=0,1, \ldots$, with limit zero as $n \rightarrow \infty$. This could be deduced from the theorem of Riemann-Lebesgue if $z^{-1} f(z)$ were shown to converge to an absolutely integrable function as $|z| \rightarrow 1-$. We show a sufficient statement that is weaker but easier to prove.

Since $f(z)$ is bounded in the sector given by $|z|<1$ and $-\tau \leq \arg z \leq \tau$, the function $F(z)$ is Lipschitz continuous there. It follows that $F\left(r e^{i \theta}\right)$ converges to a limit $F\left(e^{i \theta}\right)$, say, as $r \rightarrow 1-$, and this extension $F$ is Lipschitz continuous on the closed sector given by $|z| \leq 1$ and $|\arg z| \leq \tau$. Regard for fixed $r \leq 1$ the measure $d F\left(r e^{i \theta}\right)$ as a Lebesgue-Stieltjes measure on the interval $-\tau \leq \theta \leq \tau$ and let $G(\theta):=F\left(e^{i \theta}\right)$. For fixed $n$, the generalized Dominated Converge Theorem (Section 4 in Chapter 11 of [16]) implies that the coefficient $a_{r, n}$ converges to

$$
a_{1, n}:=\frac{1}{2 \pi i} \int_{-\tau}^{\tau} e^{-i n \theta} d G(\theta)
$$

as $r \rightarrow 1-$.

Since $F(z)$ is Lipschitz continuous as a function of $z$, the limit function $G$ is Lipschitz continuous in $\theta$. Thus, $G$ is absolutely continuous, i.e., $d G(\theta)=G^{\prime}(\theta) d \theta$ on $[-\tau, \tau]$, and the $L^{1}([-\tau, \tau])-$ norm of $G^{\prime}$ is finite, since it equals the (finite) total variation of $G$ on $[-\tau, \tau]$. The Riemann-Lebesgue theorem implies

$$
\lim _{n \rightarrow \infty} a_{1, n}=0 \text {. }
$$

We define for $|z|<1$

$$
f_{2}(z):=\sum_{n=0}^{\infty} a_{1, n} z^{n} .
$$

Let $0<R<1-\delta$. Since $F$ is Lipschitz continuous, (3.4) implies the existence of a constant $K>0$ independent of $n$ and $r$ with $\left|a_{r, n}\right| \leq K r^{-n}$. This fact together with $a_{r, n} \rightarrow a_{1, n}$ can be used to show that $f_{r, 2}$ (defined in (3.3)) converges to $f_{2}$ uniformly in $|z| \leq R$ as $r \rightarrow 1-$. It follows that $f_{r, 2}$ converges uniformly to $f_{2}$ as $r \rightarrow 1-$ in any compact subset of $|z|<1$. Clearly, $f_{r, 1}$ converges uniformly to $f_{1,1}$ in any compact subset of $\mathbb{C} \backslash \Gamma_{\tau}(\delta, 1)$.

For any $z$ inside the contour consisting of the union of $\Gamma_{\tau}(\delta, r)$ and $A_{\tau}(r)$, we have $f(z)=f_{r, 1}(z)+f_{r, 2}(z)$ by Cauchy's theorem. Letting $r \rightarrow 1-$, we obtain

$$
f=f_{1,1}+f_{2} .
$$

Let $f_{1}$ be the power series representation of $f_{1,1}$ at the origin. Since the coefficients of $f$ and of $f_{2}$ are bounded, the coefficients of $f_{1}$ are bounded as well. Moreover, for every $z$ in $A_{\tau}(1)$ except the two endpoints, $f_{1,1}$ is the analytic continuation of $f_{1}$ to a neighborhood of $z$.

Proof of Lemma 4. The proof of Szegö's theorem found in [6] on page $325 \mathrm{ff}$. can be adjusted to show Lemma 4 so we will only sketch the details. We continue to use the notation introduced before the proof of Lemma 3 , 
Assume that $g(z)=b_{0}+b_{1} z+\cdots$ is a power series with bounded coefficients which can be continued beyond the unit disk. There exist $\tau>0$ and $r>1$ so that $g$ has an analytic continuation to an open set containing the sector given by $|z| \leq r$ and $-\tau \leq \arg z \leq \tau$. Let $\varepsilon>0$. Equation (3.1) is obtained from the inequality

$$
\left|\frac{1}{2 \pi i} \int_{K_{\tau}(\delta, r)} T_{n}(z) Q^{k}\left(z^{-1}\right) \frac{d z}{z}\right|<\varepsilon,
$$

where $T_{n}(z):=b_{n}+b_{n+1} z+\cdots$ is uniformly bounded in $z$ and $n$ on $K_{\tau}(\delta, r)$ by (2) on page 325 of [6], and $Q$ is a monic polynomial that satisfies

$$
b:=\max _{z \in K_{\tau}(\delta, r)}\left|Q\left(z^{-1}\right)\right|=\max _{z \in C_{\tau}(\delta, r)}|Q(z)|<1
$$

for sufficiently small $\delta>0$ (depending on $r$ and $\tau$ ). Make $k$ so large that (3.7) is satisfied. Define $q$ to be the degree of $Q^{k}$ and $\gamma_{\nu}$ to be the coefficients of the monic polynomial $Q^{k}$. Now choose $n$ so large that (3.7) becomes (3.1) (cf. page 327 of [6] for the details).

Inequality (3.8) is obtained in 6] by using Runge's method (page 326 of 6]). Another way is to use a univalent mapping $\gamma$ from the region $|z|>1$ onto the unbounded component of $\mathbb{C} \backslash C_{\tau}(0, r)$ which maps $\infty$ to $\infty$. By Lemma 11.2 and Corollary 11.1 of Pommerenke [15], a polynomial $Q$ exists so that the value $b$ from (3.8) for $\delta=0$ is the coefficient of the linear term in the expansion $\gamma(z)=$ $b z+\kappa_{0}+\kappa_{1} z^{-1}+\cdots$. The inverse map $f$ of the function $z \mapsto 1 / \gamma(1 / z)$ is not a rotation, and $f$ can be shown to satisfy the assumptions of Schwarz' Lemma (VI.2 in [5]). Hence it satisfies $\left|f^{\prime}(0)\right|=|b|<1$. Equation (3.8) for some sufficiently small $\delta>0$ now follows from the continuity of $b$ as a function of $\delta$.

Proof of Theorem [3. Let $f(z)=a_{0}+a_{1} z+\cdots$ be a power series with bounded coefficients which is bounded in a sector. Let $f=f_{1}+f_{2}$ be the representation of $f$ from Lemma 3

Let $d>0$ be the smallest distance between any two of the (finitely many) values of $a_{n}$. Apply Lemma 4 with $g:=f_{1}$ and $\varepsilon:=d / 2$. Since the coefficients of $f_{2}$ converge to zero, the coefficients of $f$ satisfy (3.1) from some index $n_{0}$ on. We obtain numbers $\gamma_{j}$ and $q$ such that

$$
\left|\gamma_{0} a_{n}+\cdots+\gamma_{q-1} a_{n+q-1}+a_{n+q}\right|<\frac{d}{2}
$$

holds for all $n \geq n_{0}\left(q, \gamma_{0}, \ldots, \gamma_{q-1}\right)$. The fact that the coefficients $a_{n}$ are taken from a finite subset of $\mathbb{C}$ can now be used to show that the sequence $\left(a_{n}\right)$ is eventually periodic (for the details see, e.g., 6], page 327).

\section{Open PROBLEMS}

We list some of the many interesting open problems related to our results in this paper.

Question 1. What is the minimum number of zeros of modulus 1 of a reciprocal polynomial of degree $n$ with coefficients in the set $\{-1,1\}$ ?

Littlewood, [11, problem 22, poses the following research problem, which still appears to be open: "If the $n_{m}$ are integral and all different, what is the lower bound on the number of real zeros of $\sum_{m=1}^{N} \cos \left(n_{m} \theta\right)$ ? Possibly $N-1$, or not much less." 
Question 2. What is the minimum number of zeros of modulus 1 of a reciprocal polynomial with exactly $n$ nonzero terms and with coefficients in the set $\{0,1\}$ ?

Question 3. Establish whether every polynomial $p$ of degree $n$ with coefficients in the set $\{-1,1\}$ has at least one zero in the annulus

$$
\left\{1-\frac{c}{n}<|z|<1+\frac{c}{n}\right\}
$$

where $c>0$ is an absolute constant.

Question 4. S. Konyagin [10] conjectures the following for polynomials with coefficients from $\{0,1\}$ : for any fixed set $E \subset \partial D$ (the boundary of the unit disk) of positive measure there exists a constant $c(E)>0$ (depending only on $E$ ) such that for any distinct positive integers $k_{j, n}$ and any integer $n$,

$$
\int_{E}\left|\sum_{j=0}^{n} z^{k_{j, n}}\right||d z| \geq c(E) .
$$

In the same paper Konyagin shows that for each positive $\epsilon$, there exists a set $E_{\epsilon} \subset \partial D$ of measure $\pi$ and a choice of distinct positive integers $k_{j, n}$ such that

$$
\int_{E_{\epsilon}}\left|\sum_{j=0}^{n} z^{k_{j, n}}\right||d z|<\epsilon
$$

However, if his conjecture is correct, $E_{\epsilon}$ must vary with $\epsilon$.

\section{REFERENCES}

[1] P. Borwein, Computational Excursions in Analysis and Number Theory, Springer-Verlag, New York, 2002. MR1912495 (2003m:11045)

[2] P. Borwein and T. Erdélyi, Polynomials and polynomial inequalities, Springer-Verlag, New York, 1995. MR1367960 (97e:41001)

[3] P. Borwein and T. Erdélyi, Littlewood-type problems on subarcs of the unit circle, Indiana Univ. Math. J. 46 (1997), 1323-1346. MR1631600 (99j:30004)

[4] B. Conrey, A. Granville, B. Poonen, and K. Soundararajan, Zeros of Fekete polynomials, Ann. Inst. Fourier (Grenoble) 50 (2000), 865-889. MR:1779897(2001h:11108)

[5] J. B. Conway, Functions of One Complex Variable I, Springer, New York, 1995. MR.1344449 (96i:30001)

[6] P. Dienes, The Taylor Series, Clarendon Press, 1931 (reprinted by Dover Publications Inc., 1957). MR0089895 (19:735d)

[7] R. J. Duffin and A. C. Schaeffer, Power series with bounded coefficients, J. Amer. Math. Soc. 67 (1945), 141-154. MR0011322 (6:148g)

[8] T. Erdélyi, On the zeros of polynomials with Littlewood-type coefficient constraints, Michigan Math. J. 49 (2001), 97-111. MR1827077(2002c:30009)

[9] P. Erdős and P. Turán, On the distribution of roots of polynomials, Ann. Math. 51 (1950), 105-119. MR0033372(11:431b)

[10] S. Konyagin, On a question of Pichorides, C. R. Acad. Sci. Paris Sér. I Math. 324 (1997), 385-388. MR:1440953 (97k:42002)

[11] J.E. Littlewood, Some Problems in Real and Complex Analysis, D.C. Heath and Co., Lexington, MA, 1968. MR0244463(39:5777)

[12] G.V. Milovanović, D.S. Mitrinović, and Th.M. Rassias, Topics in Polynomials: Extremal Problems, Inequalities, Zeros, World Scientific Publishing, River Edge, NJ, 1994. MR.1298187 (95m:30009)

[13] A. Odlyzko and B. Poonen, Zeros of polynomials with 0,1 coefficients, Enseign. Math. (2) 39 (1993), 317-348. MR1252071 (95b:11026)

[14] Y. Peres and B. Solomyak, Approximation by polynomials with coefficients \pm 1 , J. Number Theory 84 (2000), 185-198. MR.1795789 (2002g:11107) 
[15] C. Pommerenke, Univalent Functions, Vandenhoeck and Ruprecht, Göttingen, 1975. MR0507768 (58:22526)

[16] H. Royden, Real Analysis, Macmillan Publishing Company, New York, 1988. MR.1013117 (90g:00004)

[17] G. Szegö, Tschebyscheffsche Polynome und nichtfortsetzbare Potenzreihen, Math. Ann. 87 (1922), 90-111. MR:1512103

Department of Mathematics and Statistics, Simon Fraser University, Burnaby, British Columbia, Canada V5A 1 S6

E-mail address: pborwein@cecm.sfu.ca

Department of Mathematics, Texas A\&M University, College Station, Texas 77843

E-mail address: terdelyi@math.tamu.edu

Department of Mathematics, North Dakota State University, Fargo, North Dakota 58105

E-mail address: Friedrich.Littmann@ndsu.edu 